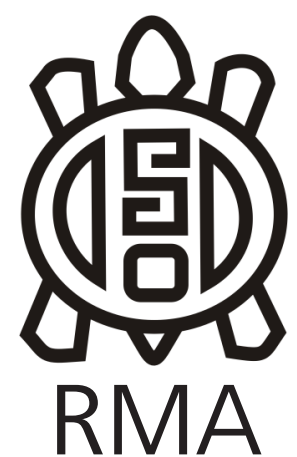

Antropología Biológica

\title{
Nuevos datos sobre salud oral y dieta en entierros secundarios del área fundacional de Mendoza (Siglos XVII-XIX)
}

\author{
New data on oral health and diet in secondary burials of the \\ foundational area of Mendoza (1 $17^{\text {th }}-19^{\text {th }}$ centuries)
}

Daniela A. Mansegosa*, Pablo S. Giannotti ${ }^{* *}$ y H. Chiavazza**

*Daniela A. Mansegosa, CONICET; Laboratorio de Arqueología Histórica, Facultad de Filosofía y Letras, Universidad Nacional de Cuyo; Centro de Investigaciones Ruinas de San Francisco, Área Fundacional, Municipalidad de Mendoza, Argentina.

E-mail: alitmansegosa@gmail.com

**Pablo S. Giannotti, CONICET; Laboratorio de Arqueología Histórica, Facultad de Filosofía y Letras, Universidad Nacional de Cuyo; Centro de Investigaciones Ruinas de San Francisco, Área Fundacional, Municipalidad de Mendoza, Argentina. E-mail: pablosebastiangiannotti@gmail.com ***Horacio Chiavazza, Laboratorio de Arqueología Histórica, Facultad de Filosofía y Letras, Universidad Nacional de Cuyo; Centro de Investigaciones Ruinas de San Francisco, Área Fundacional, Municipalidad de Mendoza, Argentina. E-mail: hchiavazza@gmail.com

\begin{abstract}
Resumen
En este trabajo se presentan los resultados del análisis de indicadores de salud oral en una muestra de cráneos y mandibulas recuperados en entierros secundarios de las Ruinas Jesuíticas de San Francisco (RSF), ubicadas en el Sitio Área Fundacional (Ciudad de Mendoza). La muestra analizada corresponde a poblaciones coloniales (siglos XVII, $X V I I I$ y XIX) y proceden de un sector de la Nave Central de las RSF. Se analizaron indicadores de salud oral y dieta: caries, desgaste dental, pérdida de dientes premortem, abscesos y periodontitis en una muestra compuesta por 200 dientes y 263 alveolos. Se analizaron las prevalencias de estos indicadores en función del sexo, edad, cronología y sector de entierro. Los resultados se integran y comparan con los obtenidos en investigaciones anteriores en muestras recuperadas de otros sectores del mismo punto arqueológico y de otros templos del Área Fundacional. Las tendencias observadas muestran diferencias en las condiciones de salud oral entre los sexos y un deterioro progresivo con el aumento de la edad, así como también diferencias entre los sectores socioeconómicos y cambios a nivel cronológico. Las prevalencias de los indicadores analizados se relacionan con una dieta mixta con una base de alimentos agrícolas ricos en carbohidratos. Esta información es complementada con datos historiográficos y arqueológicos.
\end{abstract}

Palabras clave: condiciones de vida; indicadores orales; Siglos XVII-XIX; Mendoza; arqueología histórica.

\begin{abstract}
In this paper we present the results of the analysis of oral health indicators in a sample of skulls and jaws recovered in secondary burials in the Jesuit Ruins of San Francisco (RSF), located in the. The sample analyzed corresponds to the colonial colonies (XVII - XVIII and XIX centuries) and come from a sector of the Central Ship of the RSF. The oral health and diet indicators were analyzed: caries, tooth wear, loss of premature teeth, abscesses and periodontitis in a sample composed of 200 teeth and 263 alveoli. The prevalence of these indicators was analyzed according to gender, age, chronology and burial sector. The results are integrated and compared with those obtained in previous research on samples recovered from other sectors of the same archaeological site and other temples of the Foundational Area. The observed trends differentiate between oral health conditions between the sexes and a progressive deterioration with the increase of the age, as well as between the socioeconomic sectors and a chronological level changes. The prevalences of the analyzed indicators are related to a mixed diet with a base of carbohydrate agricultural foods, this information is complemented with historiographical and archaeological data.
\end{abstract}

Keywords: living conditions; oral indicators; Centuries XVII-XIX; Mendoza; historical archeology 
Las poblaciones históricas que habitaron el norte de Mendoza durante el período comprendido entre los siglos XVI-XIX vienen siendo estudiadas desde múltiples líneas de evidencia de la cultura material (cerámica, vidrios, metales, fauna, restos vegetales, estructuras arquitectónicas, restos óseos humanos, entre otros). El objetivo general de las investigaciones se centra en comprender el proceso de conquista y colonización a través del estudio de la ciudad y los procesos socioambiantales que se dieron entorno a su fundación en el año 1561 y a su posterior consolidación (Chiavazza 2010).

El registro bioarqueológico ha aportado al estudio del estilo de vida y las condiciones de salud de las poblaciones en función de los cambios socioculturales registrados entre el período Colonial Temprano (mediados siglo XVI-XVII) y el Periodo Colonial Medio Tardío (XVIIIprincipios siglo XIX), así como también a explorar las diferencias entre los distintos sectores socio-económicos de las poblaciones que habitaron la antigua ciudad de Mendoza (Área Fundacional). En este sentido, destacan los estudios de isótopos estables (Chiavazza et al. 2015) y los de salud oral (Giannotti et al. 2017; Mansegosa 2017a) que han permitido discutir conjuntamente con información histórica la dieta y formas de procesamiento de los alimentos.

Los isótopos estables realizados en hueso de entierros humanos de distintos templos coloniales del Área Fundacional indican fuente de energía obtenida principalmente a partir de recursos C3 (trigo y cebada) (Chiavazza et al. 2015), cuestionando el consumo de maíz sostenido en las fuentes históricas. Complementariamente, los estudios arqueobotánicos destacan el temprano consumo de cereales europeos (trigo y avena), frutas (vid, olivo, durazno) y verduras (zapallo) (Chiavazza y Mafferra 2007; Mafferra 2016). El registro arqueofaunístico indica consumo de peces, vaca, chivo, cerdo, gallina (Araujo 2017; Chiavazza 2013; Ortega et al. 2006). Por otro lado, la información histórica menciona la producción local de harinas, mermeladas, vino, aguardiente, chicha, y el consumo de productos importados como el azúcar, café y chocolate (Calderón 1967; Cano Rossini 1996; Figueroa 2006). La carne vacuna se preparaba asada, hervida en puchero o en guisos junto con verduras (Acevedo 19781980; Araujo et al. 2017; Cano Rossini 1996; Coria 1988; Prieto 2000 [1983],).

El registro bioarqueológico ha permitido indagar en aspectos más específicos de las formas de consumo y acceso a los recursos por parte de los distintos sectores de la sociedad. A partir de las prevalencias de caries, se ha propuesto un consumo moderado de carbohidratos en la dieta por parte de todos los sectores de la sociedad, aunque la distribución diferencial de las lesiones podría sugerir diferencias en el tipo, calidad y procesamiento de los alimentos. Es decir, un mayor consumo de alimentos más refinados de textura más pegajosa desde edades más tempranas en aquellas personas de mayores recursos socio-económico. En cambio las lesiones registradas en las personas de menores recursos se ubican principalmente en la dentición anterior y fue interpretado como resultado del uso paramasticatorio de los dientes, sumado a otros factores (Giannotti et al. 2017). Así mismo, las prevalencias de caries en combinación con otros indicadores orales como el desgaste dental, las pérdidas de dientes premortem y los abscesos aumentan en relación con la edad, indicando un deterioro en la salud oral. También se observó un deterioro en la salud oral hacia el período Colonial Medio-Tardío, que fue interpretado como resultado de un aumento en el consumo de carbohidratos y alimentos cariogénicos. Esto se asocia con el aumento en la industria del trigo, documentado historiográficamente, y probablemente con un mayor consumo de alimentos agrícolas (Figueroa 2007; Mansegosa 2017a).

En este trabajo se presentan los resultados de múltiples indicadores de salud oral -caries, pérdida de dientes premortem, abscesos, cálculo dental y periodontitisde una muestra de cráneos, mandíbulas y dientes recuperados en entierros secundarios del templo Ruinas de San Francisco. Se evalúan estos indicadores según, sexo, edad, período cronológico y sector de inhumación, con el objetivo de evaluar las condiciones de salud oral de los individuos y contribuir a la discusión sobre posibles cambios en la dieta a lo largo del período colonial y entre los distintos sectores socioeconómicos de las poblaciones que habitaron el norte de Mendoza.

\section{Material y métodos}

La muestra seleccionada para este estudio procede de los entierros secundarios del sector de la Nave Central del templo Jesuita-Franciscano (RSF), ubicado en el Área Fundacional de Mendoza (Capital) (Figura 1). La muestra está compuesta por 200 dientes y 263 alveolos que corresponden a 14 cráneos y 8 mandíbulas aisladas (Tabla 1), los cuales constituyen todo el material dental y hueso alveolar disponible en dicho sector.

En el predio funcionaron dos templos consecutivos de origen jesuita. El primero, que funcionó entre los años 1608 y 1715 , era de menores dimensiones y fue definido arqueológicamente a partir de un piso de baldosas hexagonales (Chiavazza 2005). Los entierros humanos asignados para este templo se encuentran por debajo de los 150 centímetros de profundidad a partir del nivel de piso actual. El segundo templo era de mayores dimensiones y funcionó entre los años 1731 y 1861, año en que fue destruido por un terremoto que devastó toda la ciudad. Los entierros de este segundo templo se ubican por encima del nivel de piso del primer templo, es decir están a menos de 150 centímetros de profundidad. Las cronologías relativas asignadas a los entierros fueron realizadas por Chiavazza (2005), a partir de los pisos de 


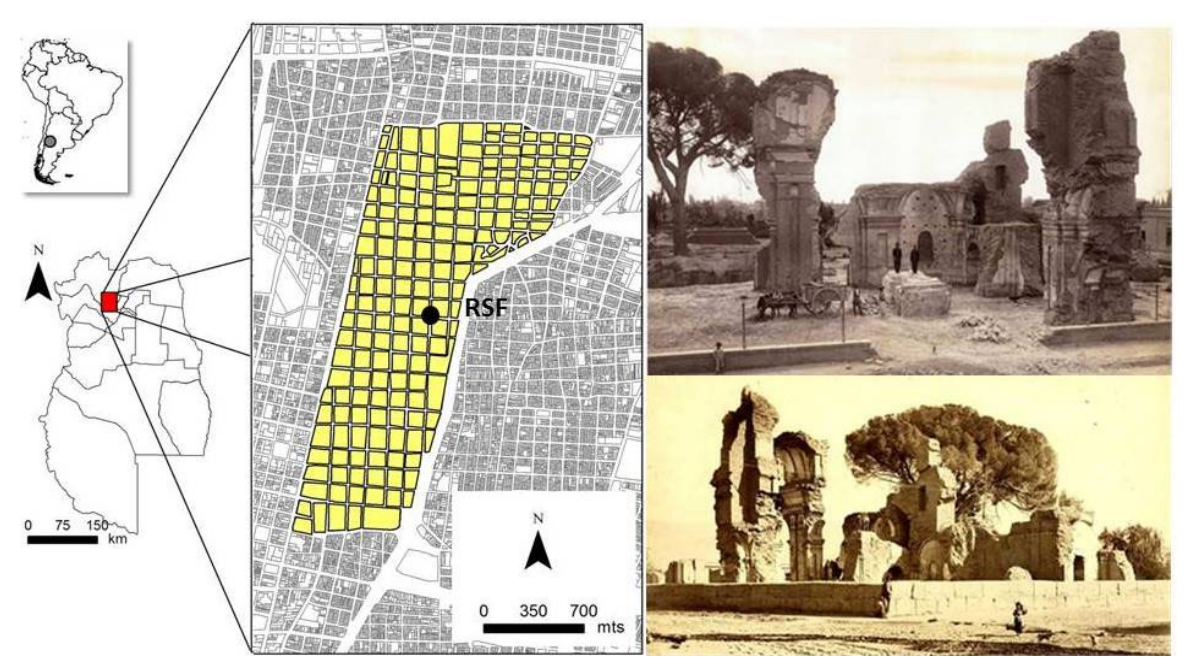

Figura 1. Ubicación del punto arqueológico RSF, el sector amarillo es el Área Fundacional de Mendoza. Las fotos de la derecha corresponden al templo San Francisco destruido por el terremoto del 20 de marzo de 1861 (Fotos del año ca. 1890)

Figure 1. Location of the RSF archaeological site, the yellow sector is the Foundational Area of Mendoza. The photos on the right correspond to the San Francisco temple destroyed by the earthquake of 20 of March of 1861 (Photos of the year ca. 1890) los templos (baldosas), ajuar mortuorio y clavazones de ataúd. En este trabajo la asignación cronológica de los elementos de la muestra se realizó siguiendo el mismo criterio, ya que fue confirmado por elementos contextuales con posibilidades de datación como clavos y tachuelas (Quiroga et al. 2017). Por lo tanto los elementos óseos y dentales fueron asignados a una cronología de acuerdo con la profundidad de entierro: se adscribieron al Colonial Temprano (CT-exterior) a aquellos hallados a más de 150 $\mathrm{cm}$. de profundidad y que corresponden a inhumaciones realizadas en el exterior del primer templo (1608 y 1715); se consideraron del período Colonial medio-tardío (CMTinterior) a aquellos que estaban por encima de los $150 \mathrm{~cm}$ de profundidad y que son inhumaciones realizadas en el interior de la segunda iglesia (1731 y 1861). Por lo tanto, cinco cráneos y dos mandíbulas son del CT-exterior; seis cráneos y tres mandíbulas corresponden al CMT-interior; y a tres cráneos y tres mandíbulas no se les pudo asignar cronología ya que no tenían referencia de profundidad de hallazgo porque fueron recuperados en excavaciones anteriores (realizadas por R. Bárcena a mediados de la década de 1990, ver Chiavazza 2005). En cuanto a la hipotética adscripción socioeconómica de los entierros, se sigue un criterio espacial ya que durante el periodo colonial, las iglesias plasmaban la segregación social en las prácticas de entierro, inhumando a los individuos de mayores recursos económicos en el interior de los templos y aquellos de menores recursos el exterior del mismo (Chiavazza 2005; De La Pena 2000).

Dado que se trata de elementos aislados y desarticulados, el sexo y la edad se establecieron considerando únicamente el cráneo y la mandíbula. Para la asignación sexual en adultos se analizó la proyección de la cresta nucal, el tamaño del proceso mastoides, el espesor del margen supraorbital y la proyección de la eminencia mentoniana (Buikstra y Ubelaker 1994); mientras que para los subadultos se consideró la prominencia mentoniana (Loth y Henneberg 2001; Schutkowski 1993). Del total de cráneos, cuatro son masculinos, cuatro femeninos y en seis no se pudo determinar el sexo por falta de conservación de las estructuras diagnósticas, por lo que se denominaron indeterminados. Las mandíbulas no pudieron ser a asignadas a ninguno de los cráneos, la mayoría ( $n: 7)$ se identificó como de sexo indeterminado y sólo una como femenino.

La estimación de la edad en adultos fue realizada considerando el cierre de las suturas, específicamente aquellas correspondientes al sistema anterior-lateral definido por Meindl y Lovejoy (1985); en subadultos se consideró el grado de formación dental (Scheuer y Black 2004). En los cráneos se identificó un (7,14\%) juvenil (12 a 20 años), 6 (42,86\%) adultos medio (35-50 años), $3(21,46 \%)$ adultos mayores (>50 años), y 4 (28,57\%) adultos indeterminados en los que no se pudo precisar la edad por mal estado de conservación de las suturas y ausencia del tercer molar, sin embargo por el grado de desgaste pudo establecerse como adulto. Las mandíbulas son todas de adulto, exceptuando una que es de un individuo juvenil.

Los indicadores de salud oral y dieta que se analizaron son las caries, la pérdida de dientes premortem (PAM), abscesos, cálculo dental, periodontitis, así como también el grado de desgaste dental. Dado que la cantidad de dientes recuperados por cráneo en muchos casos es baja y las mandíbulas no han podido asignarse a un determinado cráneo, se decidió trabajar los indicadores de salud oral por diente y alveolo, y no por individuo.

La identificación de caries se realizó macroscópicamente y fue considerada presente cuando la desmineralización formó una cavidad distintiva en el diente (Jacks y Lubell 1996, Hillson 2001). Se registró la ubicación de la lesión de acuerdo con el sistema modificado por Buikstra y Ubelaber (1994) para las lesiones en la corona y el de Hillson (2001) para aquellas ubicadas en la superficie de la raíz. Cuando había más de una lesión de caries por pieza dentaria, se registró cada una por separado. Además se calculó la Tasa Corregida de Caries (Lukacs 1995) ya que tiene en consideración la PAM.

Las PAM son aquellas pérdidas de piezas dentales 
que ocurren durante el transcurso de la vida de un individuo, relacionadas con presiones masticatorias y con la presencia de patologías orales (Lukacs 1989). Se considera que existe PAM cuando el alveolo presenta evidencias de absorción ósea; en cambio cuando no se registran evidencias de este proceso se considera pérdida postmortem. El relevamiento se realizó de acuerdo con la propuesta de Buikstra y Ubelaker (1994).

Por su parte, los abscesos son el resultado de una enfermedad pulpo-alveolar localizada, producto de la exposición, contaminación bacteriana y muerte del tejido pulpar (Ogden 2008). Se puede ocasionar a raíz de una complicación de una caries dental o cuando un diente se rompe (Hillson 2008). Siguiendo a Buikstra y Ubelaker (1994) se discriminaron los diversos tipos de abscesos según su localización (cara labial o lingual).

El cálculo dental (o sarro) es el remanente de placa bacteriana calcificado, éste se acumula en las superficies no oclusales de la corona, por encima o por debajo de la línea de las encías (Duckworth y Gao 2006). La capa de superficie que recubre contiene bacterias que promueven la inflamación oral y, en consecuencia, produce enfermedad periodontal (Hillson, 2008). Los del diente, adoptando como medida final para cada pieza la de mayor valor. De esta manera, se estableció como patológica aquellas medidas iguales o superiores a $3 \mathrm{~mm}$ (Whaites 2006).

El desgaste dental es un proceso fisiológico normal que se manifiesta en el cambio regresivo de los tejidos dentales causados por el estrés mecánico crónico (Hillson 2008). Un gran número de factores influyen en la velocidad del desgaste, entre los cuales se incluye la dureza de los alimentos, su preparación y el uso de los dientes (Minozzi et al. 2003; Deter 2009). Para el relevamiento del grado de desgaste dental en los dientes anteriores, se utilizó la propuesta de Molnar (1971), según la cual la intensidad de desgaste se registra en ocho categorías. En los dientes posteriores, se siguió el procedimiento de registro descrito por Scott (1979), que divide la superficie oclusal en cuadrantes, obtiene el valor de desgaste para cada uno de ellos utilizando una escala de 10 categorías, y luego suma los resultados para alcanzar el valor final, que va de 0 a 40. En este trabajo el grado de desgaste se agrupó en tres categorías: leve (de 0 - 3 en dientes anteriores y de 0 - 15 en posteriores), moderado (de 4 - 6 en dientes anteriores y de 16 - 30 en posteriores) y alto (de 7 - 8 en dientes anteriores y 31 - 40 en posteriores). principales factores que afectan a la prevalencia de cálculo se relacionan con las prácticas de higiene oral (Duckworth y Huntington 2006; Lieverse, 1999; Waldron, 2009). En esta investigación, el cálculo es categorizado según el método de Brothwell (1981) en tres etapas: leve (grado A), moderado (grado B) y fuerte (grado C).

La enfermedad periodontal (periodontitis), es una enfermedad lenta, progresiva y destructiva resultado de un proceso inflamatorio que es conocido como recesión alveolar (Hillson 2008). En este proceso están involucrados los ligamentos periodontales, el cemento y el hueso alveolar, dando como resultado la exposición de la raíz (Hillson 2008). Entre las causas se encuentran la gingivitis, la acumulación de placa bacteriana y la mala higiene oral (Hillson 2008; Buikstra y Ubelaker 1996). Se evaluó a partir de la medición de la distancia entre la línea amelocementaria y la cresta del hueso alveolar en las caras vestibular y lingual de todas las piezas insertas en el hueso mandibular o maxilar. Esta medición se realizó con calibre de puntas angostas y siguiendo de forma paralela el eje largo

Tabla 1. Composición de la muestra analizada

Table 1. Composition of the sample analyzed

\begin{tabular}{|c|c|c|c|c|c|c|}
\hline Código & Sexo & Edad & Cronología & Sector & $\begin{array}{c}\text { Cantidad } \\
\text { dientes }\end{array}$ & $\begin{array}{c}\text { Cantidad } \\
\text { alveolos } \\
\end{array}$ \\
\hline \multicolumn{7}{|l|}{ Cráneos } \\
\hline 19 & $M$ & A. medio & СT & Exterior & 2 & 0 \\
\hline 20 & $\mathrm{~F}$ & A. medio & СT & Exterior & 9 & 12 \\
\hline 38 & $\mathrm{~F}$ & A. indeterminado & CMT & Interior & 5 & 1 \\
\hline 234 & M & A. mayor & CMT & Interior & 0 & 15 \\
\hline 235 & $M$ & A. medio & СT & Exterior & 11 & 15 \\
\hline 236 & $\mathrm{~F}$ & A. mayor & $\mathrm{CT}$ & Exterior & 4 & 14 \\
\hline 237 & $\mathrm{~F}$ & A. mayor & CMT & Interior & 4 & 13 \\
\hline 238 & M & A. medio & CMT & Interior & 23 & 22 \\
\hline 248 & I & A. medio & СT & Exterior & 11 & 6 \\
\hline 252 & I & A. medio & CMT & Interior & 11 & 16 \\
\hline 243 & I & Juvenil & CMT & Interior & 16 & 6 \\
\hline 247 & I & A. indeterminado & IND & Indeterminado & 11 & 14 \\
\hline 249 & I & A. indeterminado & IND & Indeterminado & 5 & 16 \\
\hline $\begin{array}{l}254 \\
\text { Subtotal }\end{array}$ & $n=14$ & A. indeterminado & IND & Indeterminado & $\begin{array}{c}1 \\
113 \\
(56,5 \%)\end{array}$ & $\begin{array}{c}8 \\
158 \\
(60,1 \%)\end{array}$ \\
\hline \multicolumn{7}{|c|}{ Mandibulas aisladas } \\
\hline 242 & । & A. indeterminado & CMT & Interior & 3 & 16 \\
\hline 244 & I & A. indeterminado & CMT & Interior & 10 & 16 \\
\hline 245 & I & A. indeterminado & СT & Exterior & 16 & 11 \\
\hline 246 & I & A. indeterminado & IND & Indeterminado & 12 & 9 \\
\hline 250 & I & A. indeterminado & CMT & Indeterminado & 8 & 6 \\
\hline 253 & $\mathrm{~F}$ & A. indeterminado & IND & Indeterminado & 5 & 16 \\
\hline 255 & I & A. indeterminado & IND & Indeterminado & 16 & 14 \\
\hline $\begin{array}{l}409 \\
\text { Subtotal }\end{array}$ & $n=8$ & A. indeterminado & СT & Exterior & $\begin{array}{c}10 \\
80 \\
(40 \%)\end{array}$ & $\begin{array}{c}16 \\
104 \\
(39,5 \%)\end{array}$ \\
\hline \multicolumn{3}{|c|}{ Dientes sueltos } & - & - & $\begin{array}{c}7 \\
(3,5 \%) \\
\end{array}$ & $\begin{array}{c}1 \\
(0,4 \%)\end{array}$ \\
\hline Total & & & & & $\begin{array}{c}200 \\
(100 \%)\end{array}$ & $\begin{array}{c}263 \\
(100 \%)\end{array}$ \\
\hline
\end{tabular}




\section{Resultados}

Los resultados de los distintos indicadores de salud oral se detallan en la Tabla 2, se presentan las frecuencias y porcentajes por sexo, edad y grupo cronológico-espacial. En la Figura 2 se presentan ejemplos de los indicadores de salud oral identificados en la muestra. Del total de dientes analizados, el $8 \%$ presentó caries, mientras que el Factor de Corrección de Caries sensu Lukacs (1995) aumentó este valor resultando 10,2\%. Las lesiones se presentaron con mayor frecuencia en la dentición posterior (10\%) que en anterior (3,33\%). En general, cuando este indicador ha estado presente, se ha detectado una caries por diente y generalmente ubicada en la superficie interproximal, aunque hay casos en los que afectan la superficie oclusal y cervical. Los dientes de los hombres no registran ninguna lesión cariosa, mientras que los de las mujeres y de individuos de sexo indeterminado sí. También se observa un aumento de la cantidad de dientes con caries en relación con el incremento de la edad. A nivel cronológico-espacial se aprecia mayor porcentaje de este indicador hacia el CMT-interior, aunque los dientes indeterminados también registran altos porcentajes.

Del total de alveolos observados el 10,4\% registró PAM y el $1 \%$ abscesos. Las PAM, al igual que las caries, se presentan con mayor frecuencia en los dientes posteriores (13,3\%) que en los anteriores $(4,9 \%)$. Los abscesos se observaron en un incisivo central inferior y en dos segundos premolares (uno inferior y otro superior). Respecto del sexo, las PAM y los abscesos son más frecuentes en los hombres que en las mujeres y en los de sexo indeterminado. A medida que aumenta la edad también se incrementa la pérdida de dientes en vida. Los abscesos, por su parte, no tienen una tendencia tan clara. Respecto de la cronologíaespacialidad, al igual que las caries, las PAM son más frecuentes en el período CMT-interior que en el CT-exterior.

Los depósitos de cálculo dental se encontraban en el

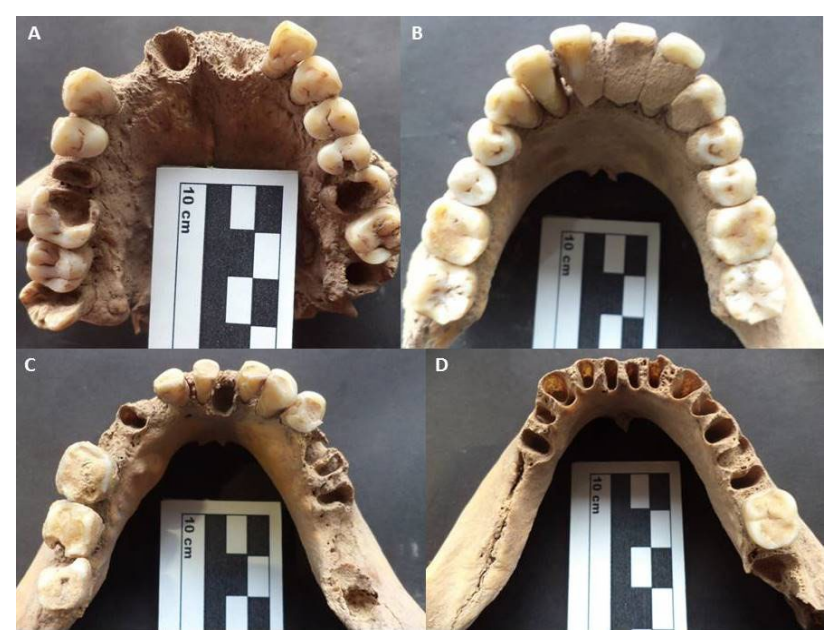

Figura 2. Ejemplos de indicadores de salud oral identificados en la muestra estudiada. A) Se observan PAM y caries en maxilar. B) Cálculo dental en dientes de mandíbula. C) PAM, caries y cálculo dental en mandíbula. D) PAM en mandíbula

Figure 2. Examples of oral health indicators identified in the sample studied. A) ATL and caries are observed in the maxilla. B) Dental calculus in jaw teeth. C) ATL, caries and dental calculus in the mandible. D) ATL in mandible

$76,5 \%$ de los dientes, repartidos de forma similar en toda la arcada (71,7\% en la dentición anterior y $71,4 \%$ en la dentición posterior). Mayoritariamente los depósitos de sarro identificados son grado $A$, es decir leve y se ubicaban en la región interproximal, aunque también habían unos pocos casos grado B y C (moderado y fuerte respectivamente) que afectaban la región bucal y lingual de las coronas dentales. En un individuo (Figura 2 C), se observaron depósitos de cálculo en la superficie oclusal de los molares lo cual se relaciona con la presencia de patologías que originarían una disfunción en la actividad masticatoria del individuo, como la pérdida de las piezas dentales antagónicas que hacen que las superficies oclusales no puedan chocar, o por una avanzada artrosis temporomandibular (Chinea et al. 1998). Las mujeres y

\begin{tabular}{|c|c|c|c|c|c|c|c|c|c|c|}
\hline & \multicolumn{2}{|c|}{ Caries } & \multicolumn{2}{|c|}{ PAM } & \multicolumn{2}{|c|}{ Abscesos } & \multicolumn{2}{|c|}{ Cálculo dental } & \multicolumn{2}{|c|}{ Periodontitis } \\
\hline & P/DO & $\%$ & P/AO & $\%$ & P/AO & $\%$ & P/DO & $\%$ & P/DO & $\%$ \\
\hline \multicolumn{11}{|l|}{ Sexo } \\
\hline $\mathrm{F}$ & $6 / 26$ & 23,1 & $6 / 56$ & 10,7 & $0 / 56$ & 0 & $22 / 26$ & 84,6 & $10 / 19$ & 52,6 \\
\hline M & $0 / 36$ & 0 & $10 / 52$ & 19 & $1 / 52$ & 1,9 & $24 / 36$ & 66,7 & $8 / 27$ & 29,6 \\
\hline I & $10 / 136$ & 7,3 & $15 / 155$ & 10 & $2 / 155$ & 1,3 & $106 / 136$ & 78 & $24 / 81$ & 29,6 \\
\hline Valor $p$ & \multicolumn{2}{|c|}{0,005} & \multicolumn{2}{|c|}{0.266} & \multicolumn{2}{|l|}{ - } & \multicolumn{2}{|c|}{0,235} & \multicolumn{2}{|c|}{0,496} \\
\hline \multicolumn{11}{|l|}{ Edad } \\
\hline Juvenil & $1 / 32$ & 3,1 & $0 / 20$ & 0 & $0 / 20$ & 0 & $29 / 32$ & 59,3 & $3 / 18$ & 16,7 \\
\hline A. medio & $4 / 67$ & 6 & $5 / 71$ & 7 & $2 / 71$ & 2,8 & $52 / 67$ & 91,2 & $18 / 49$ & 36,7 \\
\hline A. mayor & $1 / 8$ & 12,5 & $13 / 42$ & 30,9 & $1 / 42$ & 2,4 & $8 / 8$ & 100 & $7 / 8$ & 87,5 \\
\hline A. indeterm. & $10 / 91$ & 11 & $11 / 125$ & 8,8 & $0 / 125$ & 0 & $63 / 91$ & 69,2 & $11 / 53$ & 19,3 \\
\hline Valor $p$ & \multicolumn{2}{|c|}{0,411} & \multicolumn{2}{|c|}{0,001} & \multicolumn{2}{|l|}{-} & \multicolumn{2}{|c|}{0,024} & \multicolumn{2}{|c|}{0,002} \\
\hline \multicolumn{11}{|l|}{ Cronología } \\
\hline CT-Exterior & $2 / 63$ & 3,2 & $6 / 74$ & 8,1 & $0 / 74$ & 0 & $48 / 63$ & 76,2 & $11 / 44$ & 25 \\
\hline CMT-Interior & $8 / 80$ & 10 & $17 / 111$ & 15,3 & $3 / 111$ & 2,7 & $68 / 80$ & 85 & $25 / 45$ & 55,5 \\
\hline Indeterm. & $6 / 57$ & 10,5 & $8 / 91$ & 8,8 & $0 / 91$ & 0 & $28 / 57$ & 49,1 & $5 / 39$ & 12,8 \\
\hline Valor $p$ & \multicolumn{2}{|c|}{0,132} & \multicolumn{2}{|c|}{$0,432^{*}$} & \multicolumn{2}{|l|}{-} & \multicolumn{2}{|c|}{$0,011^{*}$} & \multicolumn{2}{|c|}{$0,000^{*}$} \\
\hline Total & $16 / 200$ & 8 & $31 / 297$ & 10,4 & $3 / 297$ & 1 & $153 / 200$ & 76,5 & $41 / 128$ & 32 \\
\hline
\end{tabular}

Tabla 2. Frecuencias observadas y porcentajes de cada indicador de salud oral por sexo, edad y cronología-sector. Referencias: $P$, cantidad de dientes o alveolos con presencia del indicador; DO, cantidad de dientes observados; AO, cantidad de alveolos observados. Se muestran los valores resultantes de las pruebas de significación del Test Exacto de Fisher y * $\chi^{2}$, los que están resaltados indican diferencias significativas

Table 2. Observed frequencies and percentages of each oral health indicator by sex, age and chronology-sector. References: $P$, number of teeth or socket with presence of the indicator; $O D$, number of teeth observed; $A O$, number of alveoli observed. The values resulting from the significance tests of Fisher's Exact Test and * $\chi 2$ are shown, which are highlighted indicate significant differences 
los de sexo indeterminado estaban más afectados que los hombres. Al igual que las caries y las PAM, los dientes con depósitos de cálculo dental aumentan en función de la edad y es mayor en el CMT-interior.

La periodontitis se encontró en el 32\% de los dientes. Si bien se observan en todos los dientes, es más acentuada en la región anterior $(46,7 \%)$ que en la posterior $(27,5 \%)$. Aunque se encuentra en todos los sexos, las mujeres tienen los porcentajes más altos. Como era esperable, al igual que con los indicadores anteriores, la periodontitis aumenta en relación con la edad, así como también en los entierros del CMT-interior.

Sobre los valores de prevalencia obtenidos en cada indicador de salud oral, se aplicaron pruebas estadísticas orientadas a detectar presencia de diferencias significativas entre los sexos, grupos de edad y períodos-sectores considerados (Tabla 2). Entre los sexos las diferencias entre patologías orales solo se observaron en las caries, entre los grupos de edad se detectaron diferencias significativas en una mayor cantidad de indicadores, esto es en las PAM, el cálculo dental y en la presencia de periodontitis. Se encontraron diferencias estadísticamente significativas cuando se compararon las caries entre los sexos, las PAM y la periodontitis. Entre los grupos cronológicos y de sector de entierro, las diferencias significativas se hallaron únicamente en la prevalencia de cálculo dental y en la periodontitis.

Respecto del desgaste dental, en el total de la muestra el $37,9 \%$ de los dientes presentó un desgaste leve, el 57,9\% un desgaste moderado y sólo el 4,4\% un desgaste alto. En la Figura 3 se observan los porcentajes de grado de desgaste por sexo, edad y grupo cronológico-espacial. En general las mujeres y los de sexo indeterminado tienen desgastes más variables, los hombres presentan principalmente un grado moderado. El desgaste aumenta progresivamente en relación con la edad: los adultos jóvenes predomina el desgaste leve, aunque también manifiestan un desgaste moderado; entre los adultos medio el desgaste es principalmente moderado y entre los adultos mayores es alto. A nivel cronológico- espacial, el desgaste se manifiesta en los tres grados, y parece ser más intensivo en el CMT-interior respecto del CT-exterior.

\section{Discusión}

En primer lugar, se discutirán los resultados obtenidos de los indicadores de salud oral en relación con las tendencias registradas en otras muestras del Área Fundacional, para contribuir a las discusiones sobre la dieta de las poblaciones coloniales que habitaron el norte de Mendoza. En segundo lugar, se integran los resultados de los indicadores en relación con el sexo y la edad, para evaluar diferencias en las condiciones de salud y dieta entre los sexos y los cambios en relación con el aumento de la edad. En tercer lugar, se compara y discute las

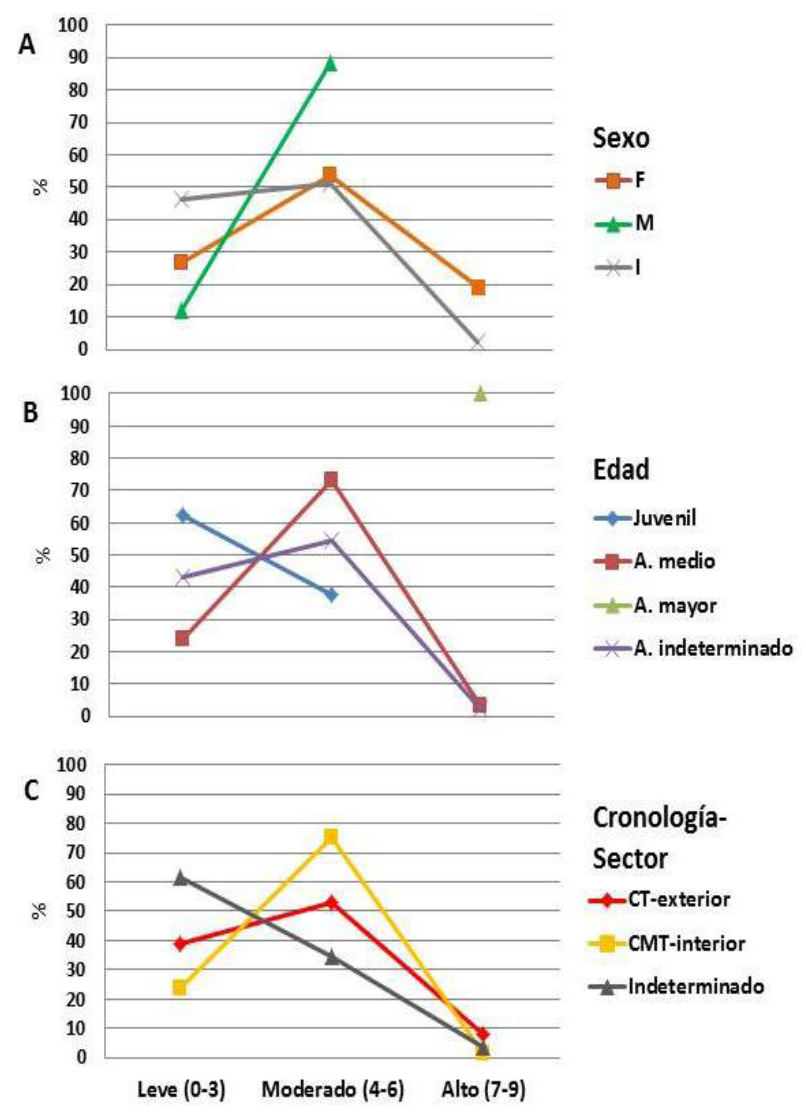

Figura 3. Grados de desgaste dental observados por sexo (A), edad (B) y sector cronología (C)

Figure 3. Degrees of dental wear observed by sex (A), age (B) and sector chronology (C)

prevalencias encontradas a nivel cronológico-espacial con los resultados de investigaciones anteriores para evaluar cambios en la salud oral y dieta a lo largo del período colonial y entre los distintos sectores socioeconómicos.

La prevalencia de caries observada en este trabajo es de $8 \%(10,2 \%$ la TCC), similar a la registrada en entierros primarios de RSF y LC que muestran un $8,7 \%$ de caries (TCC $=9,8 \%$ ) (Mansegosa 2017a), y levemente más bajo que el observado en entierros secundarios de LC que muestran 9,2\% (Giannotti et al. 2017). Es posible sugerir que los resultados obtenidos confirman las tendencias observadas en otras muestras de la población de estudio que indican un consumo moderado de carbohidratos, observado en sociedades con dietas mixtas con una base agrícola (Littleton y Frohlich 1993; Turner 1979). El registro arqueobotánico del área de estudio proporcionó evidencias del consumo de productos agrícolas de origen euroasiático como trigo, cebada, avena, centeno, vid y frutales como ciruela, manzana y durazno (Mafferra 2016). Adicionalmente, la información histórica menciona el consumo de productos agrícolas locales como el maíz e importados como el azúcar, el café y el chocolate (Acevedo 1978-1980; Cano Rossini 1996; Prieto 2000 [1983]). Por el contrario, los estudios 
isotópicos no muestran consumo de maíz, reforzando la evidencia de una dieta basada principalmente en recursos C3 (Chiavazza et al. 2015). Desconocemos si habrían consumido plantas locales silvestres como el algarrobo (Prosopis sp.), chañar (Geoffroea decorticans) y piquillín (Condalia microphylla). Las únicas evidencias del consumo de algarrobo se han registrado en áreas periféricas a la ciudad en la planicie noreste de Mendoza (Mafferra 2016). Por su parte, el registro zooarqueológico muestra evidencia de ganadería, pastoreo y consumo de aves de corral, así como también consumo de animales locales de caza y pesca (Araujo 2017; Chiavazza 2013). Todas estas evidencias indican una dieta mixta donde predomina el consumo de productos agrícolas y animales domésticos aunque se mantiene el consumo de algunos animales autóctonos. Todo esto es concordante con los índices moderados de caries dental observados en la muestra.

Respecto de la preparación de alimentos, estudios históricos mencionan que se comían verduras, cereales y carnes en forma de guisos, harinas con las que hacían el pan y mermeladas de frutas (Acevedo 1978-1980; Cano Rossini 1996; Giamportone 1998); esto da cuenta del consumo de alimentos blandos, pegajosos y ricos en carbohidratos. El uso de la técnica del hervido para la preparación de alimentos ha sido apoyada por la escasa evidencia de quemado de los huesos de los animales consumidos y el patrón de trozamiento implementado para poder ser introducidos dentro de ollas (Araujo 2017; Araujo et al. 2017). Esta técnica de preparación de los alimentos puede explicar el patrón de desgaste dental moderado observado en la mayoría de los dientes de la muestra. Otros investigadores han detectado que las tasas de desgaste dental disminuyen notablemente con el desarrollo de recipientes de cerámica para la cocción de alimentos (Molleson et al. 1993; Sciulli 1997). Por otra parte, sabemos de la existencia en la región de estudio de molinos hidráulicos para la molienda de trigo, los cuales comienzan a instalarse en el siglo XVI y aumentan en cantidad hacia el siglo XIX (Figueroa 2006). Probablemente, la incorporación de polvo y partículas minerales durante la cosecha y la molienda del trigo que se usaba para la elaboración del pan, fueron un factor importante de abrasión y por lo tanto del desgaste dental observado ${ }^{1}$. Algunos estudios apoyan esta idea dado que han atribuido el microdesgaste dental a la abrasión por fitolitos (Baker et al 1959; Walker et al. 1978), ya que son considerablemente más duros que el esmalte de los dientes.

Los depósitos de sarro dental observados en la muestra afectan a una gran cantidad de dientes $(76,5 \%)$ de una manera leve. Esto es el resultado de la mineralización de la placa bacteriana debido, fundamentalmente, al

\footnotetext{
${ }^{1}$ Recordemos que los mismos molinos se utilizaban en períodos, para molienda de material mineral procedente de precordillera (Figueroa 2008).
}

consumo de carbohidratos y a la mala higiene oral. No se han hallado evidencias de artefactos (i.e. cepillo de dientes) ni de productos para el cuidado de la higiene oral, por lo tanto, resultan esperables estos elevados índices de cálculo dental. Es importante destacar que si bien la placa se forma por la presencia de carbohidratos, ésta se mineraliza en un medio oral alcalino que se genera por una dieta rica en proteínas (Lieverse 1999), lo cual apoya el consumo de una dieta mixta. Por otra parte, en la muestra analizada se hallaron algunos dientes con mayores depósitos de sarro. Algunos autores lo han vinculado este indicador con hábitos culturales concretos como fumar tabaco (Axelsson et al. 1998; Delgado Darias et al. 2006), aunque dado su etiología multifactorial, hay que contemplar otras explicaciones como el tipo y velocidad del flujo salival (Littleton y Frohlich 1989, Whittaker et al. 1998).

Po otro lado, las PAM tienen un registro alto en las muestras observadas de 10,4\% mientras que en otras muestras del área fundacional de entierros primarios se observó 13,6\% (Mansegosa 2016). Las PAM tienen como principales etiologías la caries, la extracción para tratar el dolor producido por la lesión cariosa, la enfermedad periodontal y los traumas (Duyar y Erdal 2003; Lukacs 1992; Powell 1985). En esta muestra podemos plantear que la caries, así como también la extracción y la enfermedad periodontal pueden haber intervenido. Particularmente, sabemos en base al registro histórico que existían instrumentos para la extracción de dientes en el hospital San Antonio para la primera mitad del siglo XIX (Semorile et al. 1988).Sin embargo, para confirmar estos procedimientos de extracción de dientes será necesario realizar en futuros estudios análisis de rayos X. A su vez, la enfermedad periodontal se observó en gran parte de la muestra analizada (32\%), por lo que probablemente fue un factor que colaboró en la PAM.

A partir de la comparación e integración de las variables estudiadas podemos indagar acerca de las relaciones causa-efecto que tienen consecuencias en la salud oral y su afectación según el sexo y edad (Figura 4). El deterioro de las condiciones dentoalveolares tiene que ver con múltiples factores; por un lado, con los procesos funcionales, tales como el desgaste o fractura de la corona dental; por otro, con las características de los alimentos (tipo, dureza y textura); y por último, con la higiene bucal (Hillson 2008). De esta manera, en función de un desgaste lento o rápido según el tipo de dieta, la acumulación de placa bacteriana produce cariogénesis y enfermedad periodontal. Si el proceso avanza puede generar una infección mayor y provocar un absceso, y en última instancia, la pérdida del diente. Así mismo, la acumulación de placa bacteriana puede formar cálculo dental si se combina con una escasa higiene oral $y$, de esta manera, provocar enfermedad periodontal. Respecto de la salud oral observada entre los sexos, las mujeres tienen más caries, cálculo dental y periodontitis que los 
hombres y los de sexo indeterminado (Figura 4 A). Los hombres tienen menos caries pero tienen más PAM, por lo que las caries pueden estar subrepresentadas, dado que la caries es una de las causas más frecuentes de pérdida de dientes en vida (Hillson 2008). Asimismo, los hombres tienen menos cálculo dental y enfermedad periodontal. Dado que los estudios isotópicos realizados en muestras de entierros primarios de estas poblaciones no mostraron diferencias significativas en las dietas entre los sexos (Chiavazza et al. 2015), podemos plantear que las mujeres tienen mayores problemas de salud oral por aspectos vinculados con factores fisiológicos y/o culturales (Watson et al. 2010). Se ha demostrado que las variaciones en el flujo salival por cambios hormonales durante el embarazo, la lactancia y la menopausia pueden explicar el patrón diferencial de patologías orales (Lukacs y Largaespada 2006). Durante el embarazo, los trastornos hormonales y vasculares pueden producir una respuesta inflamatoria que afecta a la salud oral (Burakoff 2003; Laine 2002). Además, durante la menopausia los niveles de estrógeno pueden conducir a la pérdida mineral ósea y por lo tanto, este problema se puede extender al hueso alveolar y causar PAM (Hildebolt 1997; Jeffcoat et al. 2000; Laine 2002; White y Rudolph 1999; Yang et al. 2003). Contrariamente a lo hallado en la muestra estudiada, otros investigadores han registrado una mayor frecuencia de enfermedad periodontal en individuos
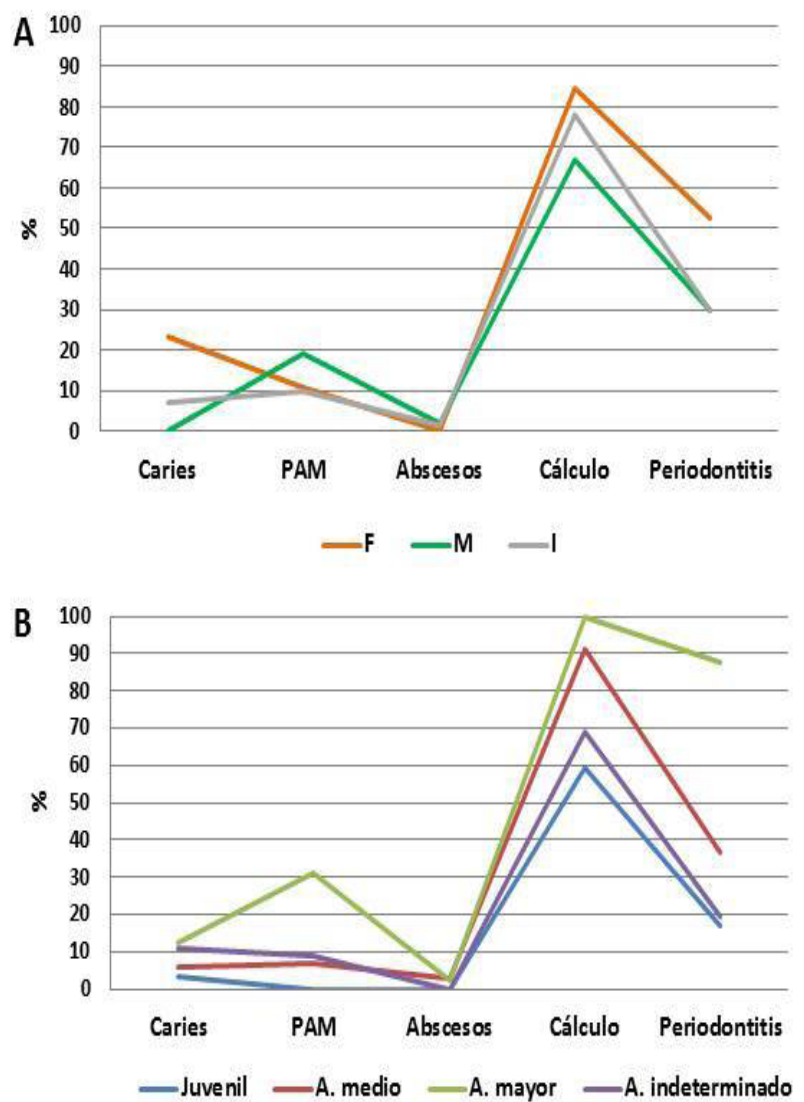

Figura 4. Integración de indicadores de salud oral según el sexo (A) y la edad (B)

Figure 4. Integration of oral health indicators according to sex $(A)$ and age $(B)$ masculinos lo cual fue interpretado como resultado de una peor higiene oral y/o una dieta diferente a la consumida por mujeres (DeWitte 2012; Wasterlain et al. 2011). Por otra parte, se ha interpretado que las mujeres tienen mayor contacto con los alimentos, resultado de la división sexual del trabajo; sin embargo entendemos que los roles de género varían entre las poblaciones. No existen datos históricos sobre este aspecto, sin embargo, se están investigando en la muestra de estudio aspectos vinculado a marcadores óseos de actividad que seguramente podrán aportar información a este respecto (Giannotti 2016). Las patologías orales son condiciones progresivas que aumentan con la edad (Delgado Darias et al. 2006; DeWitte 2012). Esta tendencia etaria general se verifica en la muestra analizada en todas las variables analizadas (excepto los abscesos) (Figura 4 B).

Por otra parte, evidencias de estrés metabólico (hipoplasias de esmalte dental), traumas en la columna (nódulos de schmorl) y osteoartrosis han mostrado la existencia de condiciones de salud y estilos de vida disímiles entre los individuos con distinto estatus socioeconómico (Mansegosa 2016; Mansegosa y Giannotti 2017). Los estudios demuestran la existencia de peores condiciones de salud en las personas de bajo estatus, lo cual refleja una sociedad estratificada con acceso diferencial a los recursos, cuidados terapéuticos y diferencias en las condiciones de actividad física. Complementariamente, los estudios osteométricos mencionan diferencias en la estatura, platimería, morfología del cráneo y de la mandíbula en relación con los grupos de estatus (Mansegosa et al. 2016). También se han evaluado cambios en las condiciones de salud a nivel cronológico, entre el CT y el CMT. Se destaca un mayor estrés metabólico durante los primeros tiempos de contacto y, por otra parte, se registra un aumento de la intensidad de la actividad física a lo largo del tiempo (Mansegosa 2017b; Mansegosa y Giannotti 2017). En la Figura 5 se observan las tendencias de salud oral entre los distintos sectores socioeconómicos (correspondientes a los sectores de entierro: interior vs. exterior) y entre los períodos cronológicos (Colonial temprano: CT vs. Colonial medio tardío: CMT). La figura compara los resultados obtenidos en este trabajo (Figura 5: NC exterior-CT y NC interior-CMT), con los resultados de investigaciones anteriores (Giannotti et al. 2017; Mansegosa 2016). Cabe aclarar, que sólo se comparan las prevalencias de caries, PAM y abscesos, ya que en los trabajos anteriores no se incluyeron datos sobre cálculo dental ni periodontitis.

En general, las muestras que corresponden a los entierros realizados en sectores de menor estatus socioeconómico (Figura 5: NC CT-exterior, Exterior RSF, La Caridad) tienen menos prevalencias de caries, de PAM, y de abscesos, es decir, mejores condiciones de salud oral que aquellos inhumados en sectores de mayor estatus (Figura 5: NC CMT-interior, RSF interior). Esto sugiere una dieta más rica en carbohidratos entre los sectores 


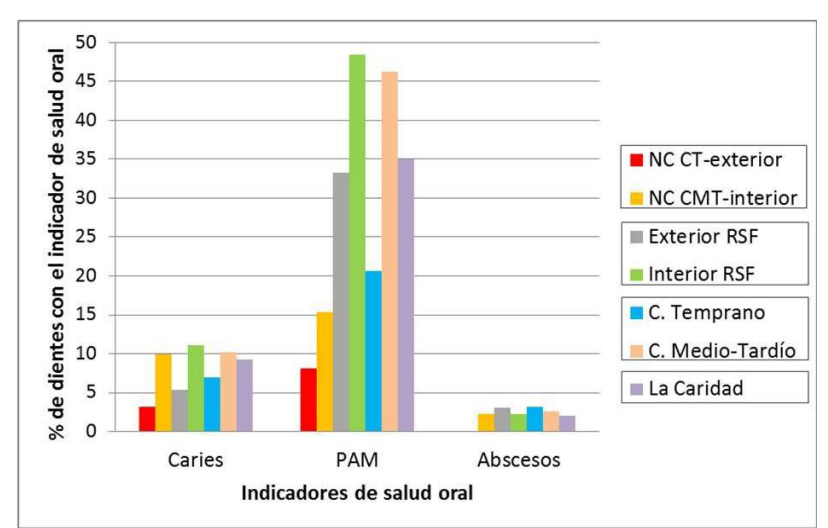

Figura 5. Comparación de prevalencias de caries, PAM y abscesos en distintas muestras coloniales del norte de Mendoza. Se comparan muestras con distinto estatus socioeconómico (estatus bajo: NC CT-exterior, Exterior RSF y La Caridad; estatus alto: NC CMT-interior, Interior RSF) y muestras de distintos períodos cronológicos (Colonial temprano: NC CT-exterior, C. Temprano; Colonial medio-tardío: NC CMT-interior, C. MedioTardío)

Figure 5. Comparison of prevalences of caries, ATL and abscesses in different colonial samples of northern Mendoza. We compare samples with different socioeconomic status (status under: NC CT-exterior, Exterior RSF and La Caridad, high status: NC CMT-interior, Interior RSF) and samples from different chronological periods (Early Colonial: NC CT-exterior, C. Early Middle Colonial: NC CMT-interior, Middle-Late C.)

más acomodados, probablemente relacionado con un mayor acceso a alimentos cariogénicos como el azúcar que, al ser importados, eran de difícil acceso. Estas posibles diferencias en la dieta deberán ser evaluadas mediante otras líneas de análisis como isótopos estables y fitolitos del tártaro dental. Las fuentes históricas también mencionan que habían harinas de trigo de distinta calidad, con las cuales fabricaban pan de dos tipos, el pan llamado "cedazo" estaba fabricado con una harina más refinada (cernida) que se vendía a un precio más alto y el llamado "de galón" de menor calidad y más barato (Figueroa 2003).

La comparación cronológica da cuenta de un aumento a lo largo del tiempo en el consumo de alimentos ricos en carbohidratos ya que las caries y las PAM aumentan (los abscesos disminuyen levemente). La evidencia arqueobotánica indica una introducción y desarrollo muy temprano de cultivos europeos (trigo, vid, olivo, etc) que se dio con el objetivo de asegurar el éxito de la conquista española y posteriormente la consolidación de la ciudad (Mafferra 2016). Asimismo, los registros históricos mencionan molinos hidráulicos para moler el trigo desde el siglo XVI con un incremento hacia mediados del siglo XIX. Por lo tanto, podemos inferir que estos bioindicadores de salud oral sugieren un consumo de pan de trigo y de productos agrícolas desde los primeros momentos del contacto hispano-indígena, y probablemente una mayor dependencia de estos hacia el período CMT.

\section{Consideraciones finales}

En este trabajo, hemos aportado nuevos datos para el estudio de la salud oral de las poblaciones coloniales que habitaron el norte de Mendoza. Hemos demostrado que las mujeres tenían mayores problemas de salud oral que los hombres probablemente causados por actividades vinculadas a los roles de género, así como también por factores fisiológicos relacionados con la biología reproductiva. Todos los indicadores analizados mostraron un aumento en relación con la edad lo que da cuenta de la progresividad de los procesos patológicos y funcionales propios del uso del aparato masticatorio.

Se observaron algunas diferencias de salud oral entre los sectores socioeconómicos, que indican mayores problemas entre los individuos enterrados en espacios de mayor estatus, probablemente vinculado a un mayor acceso a alimentos cariogénicos (i.e. azúcar) y a distintos métodos de procesamiento de alimentos (i.e. mayor refinamiento de harinas). Las evidencias de caries, PAM y abscesos indican que el consumo de alimentos cariogénicos estuvo presente durante todo el período colonial, incluso desde épocas muy tempranas. Dichos indicadores muestran un aumento a lo largo del tiempo, por lo tanto sugieren un cambio en la dieta hacia una mayor dependencia en alimentos de origen vegetal ricos en carbohidratos. Si bien, los valores obtenidos apoyan las tendencias observadas en trabajos anteriores, son necesarias nuevas líneas de evidencia tales como estudios isotópicos y análisis de fitolitos contenidos en el tártaro dental.

\section{Agradecimientos}

A los investigadores y estudiantes que integran el Centro de Investigaciones Ruinas de San Francisco que colaboraron en las excavaciones de las muestras analizadas y al Área Fundacional de la Municipalidad de Mendoza. Esta investigación se financió con una beca postdoctoral otorgada por el Consejo Nacional de Investigaciones Científicas y Técnicas a uno de los autores (DAM) y con un subsidio de la Agencia Nacional de Promoción Científica y Tecnológica-UNCuyo (PICTO 2016-0045).

\section{Bibliografía}

Acevedo, E. O. 1978-1980. El abastecimiento de Mendoza, 1561-1810. Revista de Historia Americana y Argentina 19-20: 9-33.

Araujo, E. J. 2017. Prácticas alimentarias en el período prehispánico tardío y Colonial temprano: estudio de un contexto doméstico en las ruinas de San Francisco (Valle de Mendoza, Siglos XV-XVI). Revista de Arqueología Histórica Argentina y Latinoamericana. En prensa.

Araujo, E., L. Castillo, H. Chiavazza y C. Prieto. 2017. 
Cocinar y alimentarse en tiempos de conquista. Reconstruyendo paquetes culinarios a partir de análisis cerámicos y arqueofaunísticos (Mendoza, siglos XVXVII). Estudios Atacameños. En prensa.

Axelsson, P., J. Paulander y J. Lindhe. 1998. Relationship between smoking and dental status in 35-50-6 and 75 year old individuals. J. Clin. Periodontol. 25, pp. 297-305.

Baker, G., J. H. P. Jones y I. D. Wardrop. 1959. Cause of wear in sheep's teeth. Nature 184:1583-1584.

Brothwell, D. R. 1981. Digging up bones. the excavation, treatment and study of human skeletal remains ( $3^{\circ} \mathrm{ed}$.). New York. Cornell University Press.

Buikstra, J. E. y D. H. Ubelaker. 1994. Standards for Data Collection from Human Skeletal Remains. Arkansas. Archaeological Survey Research Series N 44.

Burakoff, R. P. 2003. Preventive dentistry: current concepts in women's oral health. Primary Care Update OB/GYNs 10:141-146.

Calderon, J. L. M. 1967. Mendoza hace cien años. Historia de la provincia durante la presidencia de Mitre. Buenos Aires. Ediciones Teoría.

Cano Rossini, L. 1996. La mujer mendocina de 1800. Una Revolución cultural en marcha. Mendoza. Ediciones Culturales de Mendoza.

Chiavazza, H. 2005. Los templos coloniales como estructuras funerarias. Arqueología en la iglesia jesuita de Mendoza. Londres. British Archaeological Reports. International Series 1388.

Chiavazza, H. 2010. Procesos sociales y ambientales en el sector urbano de Mendoza entre los siglos XV-XVIII: arqueología urbana e historia ambiental. Comechingonia virtual 4 (2): 227-253.

Chiavazza, H. 2013. No tan simples: pesca y horticultura entre grupos originarios del Norte de Mendoza. Comechingonia virtual 1: 27-45.

Chiavazza, H., D. Mansegosa y A. Gil. 2015. Human diet and residential mobility in the Central Western Argentina colony: stable isotopes (13C, 15N, 180) trends in archaeological bone samples. International Journal of Historical Archaeology 19 (2): 289-308.

Chiavazza, H. y L. Mafferra. 2007. Estado de las investigaciones arqueobotánicas en Mendoza y sus implicancias en la arqueología histórica. Revista de arqueología histórica Argentina y Latinoamericana 1: 127-152.

Coria, L. A. 1988. Evolución económica de Mendoza en la época colonial. Mendoza. Ediciones Culturales de Mendoza.

Delgado Darias, T., J. Velasco Vázquez, M. Arnay de la Rosa, E. Martín Rodríguez y E. González-Reimers. 2006. Calculus, periodontal disease and tooth decay among the prehispanic population from Gran Canaria. Journal of Archaeological Science 33: 663-670.

Deter, C. 2009. Gradients of occlusal wear in huntergatherers and agriculturalists. American Journal of Physical Anthropology 138: 247-254.

DeWitte, S. N. 2012. Sex differences in periodontal disease in catastrophic and attritional assemblages from medieval London. American Journal of Physical Anthropology 149:405-416

Duckworth, R. M. y X. J. Gao. 2006. Plaque as a reservoir for active ingredients. En: R. M. Duckworth (ed.). The Teeth and Their Environment, pp. 132-149. Karger, Basel. Monographies in Oral Science 19.

Duckworth, R. M. y E. Huntington. 2006. On the relationship between calculus and caries. En: R. M. Duckworth (ed.). The Teeth and Their Environment, pp. 1-28. Karger, Basel. Monographies in Oral Science 19.

Duyar, I. y Y. S. Erdal. 2003. A new approach for calibrating dental caries frequency of skeletal remains. Homo 54:57-70.

Figueroa, P. 2003. Introducción a la historia alimentaria de Mendoza. Cuadernos del Centro De Graduados de la Facultad De Filosofía y Letras- UNCuyo 101-121.

Figueroa, P. 2006. Los molinos hidráulicos en Mendoza (Argentina) durante el período colonial (S. XVI, XVII y XVIII). Revista Universum 21 (1): 1-24.

Figueroa, P. 2007. Trapiches e ingenios mineros en la Mendoza Colonial. Tiempo y espacio 20: 84-97.

Giamportone, T. A. 1998. Mendoza a través de los viajeros 1820-1850. En: Cueto, A. y V. Ceverino (eds.) Los Hombres y Las Ideas en la Historia de Cuyo, pp. 193-213, Tomo II. Mendoza. Facultad de Filosofía y Letras de la Universidad Nacional de Cuyo.

Giannotti, P. S. 2016. Marcadores de estrés ocupacional en poblaciones históricas del norte de Mendoza (s. XVI-XIX): primeros resultados exploratorios. Comechingonia Revista de Arqueología 20 (1): 81-100.

Giannotti, P. S., D. A. Mansegosa y H. Chiavazza. 2017. Caries dental y salud oral en poblaciones coloniales de Mendoza (Argentina) durante los siglos XVIII-XIX. Estudios Atacameños. En prensa. 
Hildebolt, C. F. 1997. Osteoporosis and oral bone loss. Dentomaxillofacial Radiology 26:3-15.

Hillson, S. 2001. Recording dental caries in archaeological human remains. International Journal Osteoarchaeology 11: 249-289.

Hillson, S. 2008. The current state of dental decay. En: J. D. Irish, J. D. y G. C. Nelson (eds.) Technique and application in dental anthropology, pp. 111-135, Cambridge University Press.

Jackes, M. y D. Lubell. 1996. Dental pathology and diet: second thoughts. En: Otte, M (ed.) Nature et culture: Actes du Colloque International de Lie'ge, pp. 68: 457480 Etudes et Recherches Arche'ologiques de L'Universite' de Lie'ge.

Jeffcoat, M. K., C. E. Lewis, C. Y. Reddy Ms Wang y M.A. Redford. 2000. Postmenopausal bone loss and its relationship to oral bone loss. Periodontology 23:94-102.

Laine, M. A. 2002. Effect of pregnancy on periodontal and dental health. Acta Odontologica Scandinavica 60:257-264.

Lieverse, A. 1999. Diet and the aetiology of dental calculus. International Journal of Osteoarchaeology 9: 219-232.

Littleton, J. y B. Frohlich. 1989. An analysis of dental pathology and diet in historic Bahrein. Paleorient 15:59-84.

Littleton, J. y B. Frohlich. 1993. Fish-eaters and farmers: dental pathology in the Arabian Gula. American Journal of Physical Anthropology 92: 427-447.

Loth, S. y M. Henneberg. 2001. Sexually dimorphic mandibular morphology in the first few years of life. American Journal of Physical Anthropology 115: 179-186.

Lukacs, J. R. 1989. Dental Paleopathology: Methods for Reconstructing Dietary Patterns. En: Yasar Iscan, M. y K. A. R. Kennedy (eds.) Reconstruction of Life from the Skeleton, pp. 261-286. New York. Wiley-Liss.

Lukacs, J. R. 1992. Dental pathology and agricultural intensification in South Asia new evidence from Bronce Age Harappa. American Journal Physical Anthropology 87: 133-150.

Lukacs, J. R. 1995. The 'Caries Correction Factor': A New Method of Calibrating Dental Caries Rates to Compensate for Antemortem Loss of Teeth. International Journal of Osteoarchaeology 5:151-156.

Lukacs, J. y L. Largaespada. 2006. Explaining Sex Differences in Dental Caries Prevalence: Saliva, Hormones, and "Life-History" Etiologies. American Journal of Human
Biology 18: 540-555.

Mafferra, L. 2016. Arqueobotánica del norte de Mendoza: interpretaciones sobre el rol de los vegetales en la interacción indígena-hispana durante los siglos XVI y XVII. Serie Publicaciones del CIRSF, 9. Facultad de Filosofía y Letras de la Universidad Nacional de Cuyo. Mendoza.

Mansegosa, D. A. 2016. Estudios sobre salud y enfermedad en poblaciones históricas urbanas de Mendoza (Argentina). Nuevos aportes para el estudio bioarqueológico de La Caridad. Comechingonia virtual Revista de Arqueología 20 (1): 111-142.

Mansegosa, D. A. 2017a. Dieta y condiciones de salud oral en poblaciones históricas del norte de mendoza. Revista de Arqueología Histórica Argentina y Latinoamericana. En prensa.

Mansegosa, D. A. 2017b. Hipoplasias de esmalte dental y estrés metabólico en poblaciones del norte de Mendoza (Argentina): siglos XVII-XIX. Intersecciones en Antropología 18: 197-207.

Mansegosa, D. A. y P. S. Giannotti. 2017. Los nódulos de Schmorl y sus implicancias en la salud de una población histórica colonial (Mendoza, Argentina). Revista Anales de Arqueología y Etnología. En prensa.

Mansegosa D. A, L. P. Menéndez y P. S. Giannotti. 2016. Variación morfológica craneofacial y mandibular en poblaciones históricas de Mendoza: Siglos XVII-XIX. XXI Congreso Nacional de Arqueología Argentina 1337- 1343.

Meindl, R. S. y C. O. Lovejoy. 1985. Ectocranial suture closure: a revised method for the determination of skeletal age at death based on the lateral-anterior sutures. American Journal of Physical Anthropology 68:57-66.

Minozzi, S., G. Manzi, F. Ricci, S. di Lernia y S. Borgognini Tarli. 2003. Nonalimentary tooth use in Prehistory: an example from Early Holocene in Central Sahara (Uan Muhuggiag, Tadrart Acacus, Libya). American Journal of Physical Anthropology120: 225-232.

Molleson, T., K. Jones, S. Jones. 1993. Dietary change and the effects of food preparation on microwear patterns in the late Neolithic of Abu Hureyra, northern Syria. Journal of Human Evolution 24:455-468.

Molnar, S. 1971. Human tooth wear, tooth function, and cultural variability. American Journal of Physical Anthropology 34:175-190.

Ogden, A. 2008. Advances in the palaeopathology of teeth and jaws. En Pinhasi, R. y S. Mays (eds.) Advances in human palaeopathology, pp. 283-307. Chichester, England. Wiley. 
Ortega, C., F. Hernández y D. Barboza. 2006. Estudios zooarqueológicos del predio mercedario del área fundacional de Mendoza. En Chiavazza H. y V. Zorrilla (eds.) Arqueología en el predio mercedario de la ciudad de Mendoza, pp. 79-120. Mendoza. Facultad Filosofía y Letras de la Universidad Nacional de Cuyo.

Powell, M. L. 1985. The analysis of dental wear and caries for dietary reconstruction. En: Gilbert R y J. H. Mielke (eds.) The analysis of prehistoric diets, pp. 307-339. Orlando. Academic Press.

Prieto, M. R. 2000 [1983]. Formación y consolidación de una sociedad en un área marginal del Reino de Chile: la Provincia de Cuyo en el siglo XVII. Tesis doctoral, Universidad de Sevilla. Anales del Instituto de Arqueología y Etnología 52-53: 18-366.

Quiroga, M., H. Chiavazza, G. Lascalea y M. Gurrito. 2017. Estudios arqueométricos de clavos procedentes de contextos funerarios de las Ruinas de San Francisco (Mendoza, Argentina). 2017. Arqueología 23 (2) 1-16.

Scheuer, L. y S. Black. 2000. Developmental Juvenile Osteology. London. Academic Press.

Schutkowski, H. 1993. Sex determination of infant and juvenile skeletons: I. Morphognostic features. American Journal of Physical Anthropology 90: 199-205.

Sciulli, P. W. 1997. Dental evolution in prehistoric native Americans of the Ohio Valley Area. I. Wear and pathology. International Journal of Osteoarchaeology 7:507-524.

Scott, E. C. 1979a. Dental wear scoring technique. Am J Phys Anthropol 51:213-218.

Semorile, A., E. Martín De Codoni, L. Cano Rossini, M. Puig Lopez y S. Laria. 1988. Historia de la medicina de Mendoza. Tomo I y II. Mendoza.
Turner, C.G II. 1979. Dental anthropological indications of agricultura among the Jomon people of central Japan. American Journal Physical Anthropology 51: 619-636.

Waldron, T. 2009. Paleopathology. Cambridge. Cambridge University Press.

Walker, A. C, H. N. Hoeck y L. Perez. 1978. Microwear of mammalian teeth as an indicator of diet. Science 201:908-910.

Wasterlain, S. N., E. Cunha y S. Hillson. 2011. Periodontal disease in a Portuguese identified skeletal sample from the late nineteenth and early twentieth centuries. American Journal of Physical Anthropology 145:30-42.

Watson, J. T., B. T. Arriaza, V. Standen e I. Muñoz. 2010. Occlusal Dental Wear and the Formative Transition along the Northern Chilean Coast. American Journal of Osteoarchaeology 23: 287-302.

Whaites, E. 2006. Essentials of Dental Radiography and Radiology ( $4^{\circ}$ ed.). Dental Series. Edinburgh: Churchill Livingstone.

White, S. C y D.J. Rudolph. 1999. Alterations of the trabecular pattern of the jaws in patients with osteoporosis. Oral Surgery Oral Medicine Oral Pathology Oral Radiology 88:628-635.

Whittaker, D. K, T. Molleson y T. Nuttall. 1998. Calculues deposits and bone loss on the teeth of Romano- British and eighteenth-century Londoners. Archives of oral biology 43: $941-948$.

Yang, J., S. M. Pham, D. L. Crabbe. 2003. Effects of estrogen on rat mandibular and tibial microarchitecture. Dentomaxillofacial Radiology 32:247-251. 